\title{
Development of TNU-SuperSID Teaching Module for Observing the Effects of Solar Activities on the Lower lonosphere
}

\author{
Le Minh Tan \\ Department of Physics, Faculty of Natural Science and Technology, Tay Nguyen University, Buon Ma Thuot 63000, Vietnam
}

\begin{abstract}
Department of Physics, Faculty of Natural Science and Technology, Tay Nguyen university has collaborated with Stanford Solar Center of Stanford university on Space Weather monitor project. We have developed the TNU-SuperSID teaching module which has three main parts: antenna, preamplifier and data logger. This module can detect the variation of Very Low Frequency (VLF, $3-30 \mathrm{kHz}$ ) signals during sunrise and sunset transition, and sudden ionospheric disturbance due to solar flares. In other word, the behavior of the Earth's lower ionosphere corresponding the solar activities is understood by using VLF technique. Our project helps undergraduate students who are learning the astrophysics and space physics to enhance their knowledge in space science and their technical skills with real experiments. Through the participant in this project, students can also be gained their skills such as communication, working in team, processing data, etc.
\end{abstract}

Key words: TNU-SuperSID teaching module, lower ionosphere, solar activities, space science, technical skills.

\section{Introduction}

Stanford Solar Center, Electrical Engineering Department of Stanford University, USA has developed an inexpensive monitor, SuperSID, which students at High Schools and universities around the world can use to track changes of the Earth's ionosphere. This version is the product of the Space Weather Monitor project, which satisfies the goal of IHY (International Heliophysical Year) that is to advance the understanding of basic heliophysical processes that govern the Sun, Earth and heliosphere and to illustrate the beauty, relevance and significance of space and Earth science to the world [1]. SuperSID monitor can detect the SID (Sudden Ionospheric Disturbance) due to solar flares by recording the Very Low Frequency (VLF, $3-30 \mathrm{kHz}$ ) signals emitted from many transmitters to a receiver. Especially, students can base on the standard Stanford's circuit to assemble the preamplifier and to

Corresponding author: Le Minh Tan, research fields: space physics and electronics. E-mail: tantaynguyen82@yahoo.com. build their own antenna [2]. Since 2011, Department of Physics, Faculty of Natural Science and Technology, TayNguyen University, Vietnam has collaborated with Stanford Solar Center for SuperSID monitor program, and Tay Nguyen University's VLF team have been working in the VLF field and sharing scientific data with other worldwide teams.

After the success of the IHY program, the ISWI (International Space Weather Initiative) was established that mainly works in a scientific field called "Space Weather". The Space Weather concept is defined as conditions on the Sun, solar wind, magnetosphere, the Earth's ionosphere, and the neutral atmosphere that can affect the performance and reliability of space - borne and ground- based technological systems and endanger human life or health. Space Weather also spans other large fields including solar physics, plasma physics, and stellar astronomy [3]. The ionospheric studies serving the prediction of Space Weather become imperative. Our 
department also participated in ISWI program, and a SuperSID version supported by Stanford University has been operating for two years. In order to continuously collaborate with Stanford University, we design the SuperSID teaching module for undergraduate-student education and for research activities at Tay Nguyen University.

This paper aims to report the development of TNU-SuperSID teaching module and give some examples of experimental results to illustrate the ability of this module. In addition, we propose the educating process to teach students who are studying in Astrophysics and Atmospheric Physics subjects. Our project's purpose is to help students not only to develop their technical skills, but also to understand the fundamental behavior of the lower ionosphere corresponding to activities of the Sun.

\section{Background of the Relationship between the Earth's Ionosphere and the Sun's Activities}

The Earth's ionosphere is the top part of the atmosphere with an altitude of $60 \mathrm{~km}$ to $1,500 \mathrm{~km}$. Energy in the solar radiation ionizes gas molecules such as $\mathrm{N}_{2}, \mathrm{O}_{2}$, and $\mathrm{O}$ into positive ions and free electrons. The free electrons generated during this process can greatly affect the transmission of radio signals [4].

The ionosphere is divided into different layers. The number of layers of the ionosphere varies from day night due to the effects of the Sun's activities. During the day, the ionosphere is ionized by strong solar radiation, which forms many different layers including $\mathrm{D}, \mathrm{E}$ and $\mathrm{F}$. The D layer is the lowest layer and has the complex chemistry processes. The top layer is $\mathrm{F}$, including F1 and F2 layers. Based on the wavelength, the spectrum of solar radiation is divided into three groups, (a) < $14 \mathrm{~nm}$, (b) 14-80 nm, and (c) 80-102.7 $\mathrm{nm}$. The electrons in the E layer are formed by the wavelength of the radiation group (a) and (c) ionizing the $\mathrm{N}_{2}, \mathrm{O}_{2}$ and $\mathrm{O}$. Meanwhile, the electrons in the $\mathrm{F}$ layer are formed by the radiation group (b) ionizing the $\mathrm{N}_{2}$ and $\mathrm{O}$ [5].

In daytime, the reflection height of VLF wave mainly depends on the solar zenith angle according to Chapman's principle [6]. The VLF signal strength will be "smooth" and reach to maximum value at noon. At night, when the D layer almost disappears, the VLF waves are reflected at E layer with altitude about $90 \mathrm{~km}$ and the lowest layer of the ionosphere makes signal's strength to be more unstable than that in daytime conditions [7].

It is well known that solar flares cause the perturbations of the VLF signals propagating in the Earth - Ionosphere waveguide with the early work of Mitra (1974). In day time, in normal conditions, the D layer is maintained by ionization factor, Lyman- $\alpha$ radiation $(121.6 \mathrm{~nm})$ from the Sun and VLF waves reflected at an altitude about $70 \mathrm{~km}$ [5]. In normal conditions, X-rays radiated from the Sun affect the $\mathrm{D}$ layer insignificantly. When the solar flares occur, the strong ionizing radiation causes the electron density of the D layer to dramatically increase with 1-2 orders of magnitude, and hence the sudden changes of amplitude and phase of the VLF signals are found at the VLF receivers. This phenomenon is known as SID (Sudden Ionospheric Disturbance) [8, 9]. The X-ray intensity of solar flares recorded by GOES (Geostationary Operational Environmental Satellites) is divided into five levels: A, B , C , M , X. Among them, A class is the lowest class while $\mathrm{X}$ class is the highest class. Each class is divided into different levels from 1.0 to 9.9 [10].

During solar flares, particles such as electrons, protons, etc. are ejected from the Sun. The plasma clouds consisting of those particles are called CME (Coronal Mass Ejection). It would take a CME approximately $72 \mathrm{~h}$ to reach the Earth [2]. These matters cause ionospheric storms which disrupt satellites and communications; magnetic storms that damage currents in power grids; as well as radiation storms that endanger human lives [3]. 


\section{Development of TNU-SuperSID Teaching Module}

TNU-SuperSID teaching module is developed with a magnetic antenna, a preamplifier, a sound card, a PC and Stanford University’s recording software.

\subsection{Development of VLF Antenna}

The VLF antenna of TNU-SuperSID teaching module records the magnetic components of electromagnetic waves. This antenna consists of a frame and wire loops. The frame made of PVC is a square with the base of $1 \mathrm{~m}$ (Fig. 1a). The copper wires with the size of 24 AWG (wire's diameter is $0.511 \mathrm{~mm}$ ) are used to assemble the VLF antenna (Fig. 1b). The frame is wrapped with 20 turns of that wire (Fig.1c). The impedance of antenna is $5.7 \Omega$ and the inductance is $50 \mathrm{mH}$. The voltage will be induced in the loop if the magnetic field of the electromagnetic waves changes and passes the antenna. The output voltage is given by Faraday's law [11]:

$$
V=j 2 \pi f N A B \cos \theta
$$

where $V$ is the output voltage, $N$ is the number of turns in the antenna, $A$ is area of antenna (in $\mathrm{m}^{2}$ ), $B$ is the magnetic flux density (in $\mathrm{T}$ ), and $\theta$ is the angle of the magnetic field from the axis of the loop. The response pattern of the antenna is a dipole in azimuth because the axis of the loop is horizontal.

The normalized antenna sensitivity is obtained [11],

$$
S_{O}=\frac{c\left(4 k T R_{a}\right)^{\frac{1}{2}}}{2 \pi N A}
$$

where, $A=1 \mathrm{~m}^{2}$ is area of loop. $R_{a}=5.7 \Omega$ is the loop
DC resistance, $N=20$ is the number of turns, $c=$ $2.998 \times 10^{8} \mathrm{~ms}^{-1}$ is the speed of light, $k=1.38 \times 10^{-23} \mathrm{JK}^{-1}$ is Boltzmann's constant, $T$ is the temperature in Kelvins. At room temperature, we have $4 k T=1.61 \times$ $10^{-20}$ joule. Therefore, the normalized sensitivity of this antenna is $7.31 \times 10^{-4} \mathrm{~V} / \mathrm{Hz}^{-1 / 2} \mathrm{~m}^{-1}$.

To avoid electromagnetic interference and resonance, the antenna is installed far from the places where has the generators, electrical equipments and power lines.

\subsection{Development of VLF Amplifier}

The preamplifier filters and amplifies the small signals for digitization by the ADC (analog to digital converter). The amplified signals are transmitted by a long coaxial cable to the PC.

In addition, the circuit layout is designed by using Altium software, and then the double layer PCB (printed circuit board) is also made. Fig. 2a and 2b show the circuit layout viewed in the software and PCB layout. Fig. 2c shows the PCB after being assembled with the electronic components. Finished board is enclosed in the plastic box.

Furthermore, a generator is used to provide the input of amplifier with the sine signal of $48 \mathrm{mV}$ and the frequency from $100 \mathrm{~Hz}$ to $100 \mathrm{kHz}$. The oscilloscope is used to record the output signal. First, output voltages are measured, then the gain of amplifier in decibel $(\mathrm{dB})$ is calculated and the variation of gain values versus the frequency is plotted. Fig. 3 shows that the $3 \mathrm{~dB}$ points appear at $\sim 700 \mathrm{~Hz}$ on the low side and at $\sim 20 \mathrm{kHz}$ on the high side.

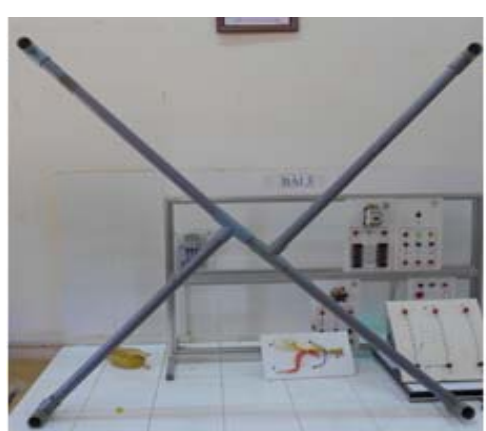

(a)

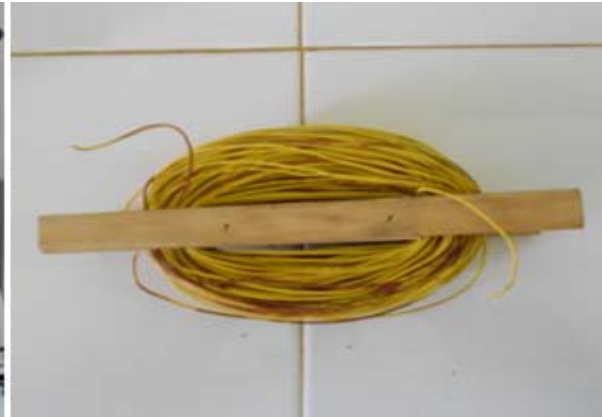

(b)

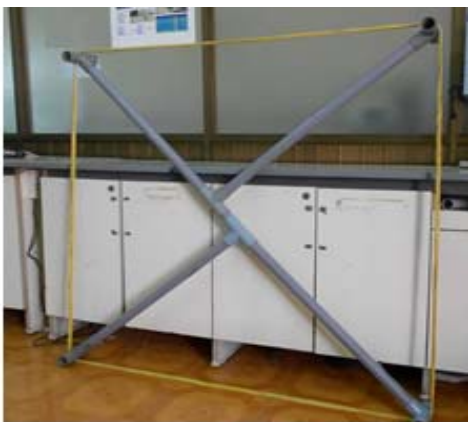

(c)

Fig. 1 The process of assembling the VLF antenna. 


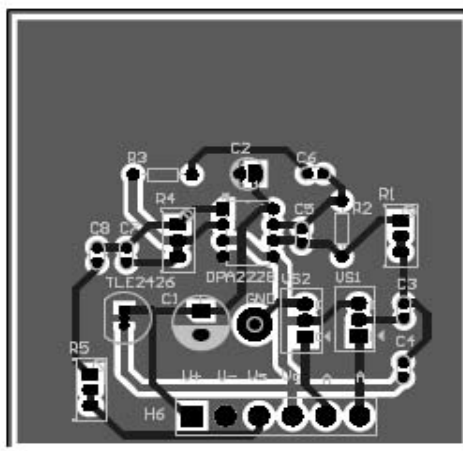

(a)

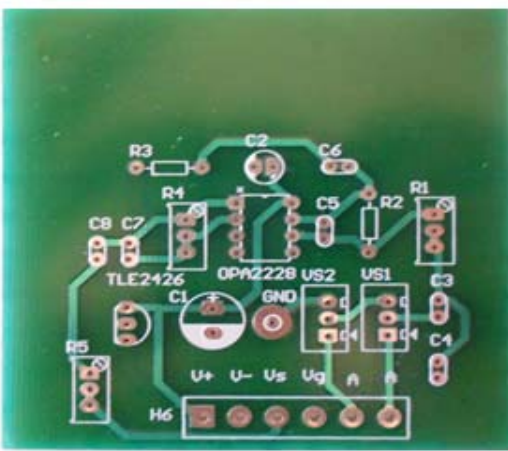

(b)

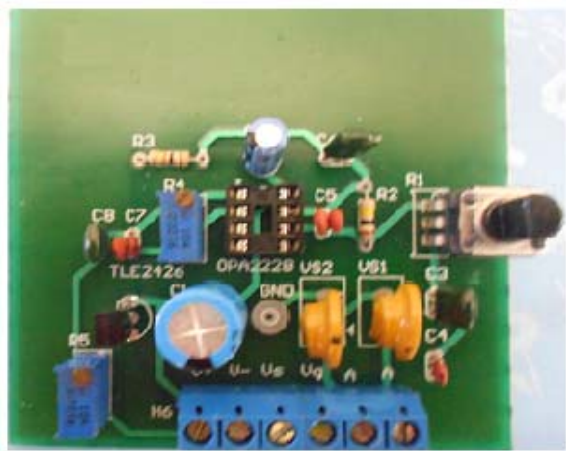

(c)

Fig. 2 a) circuit layout on software, b) PCB before assembly and c) PCB after assembled with electronic components.

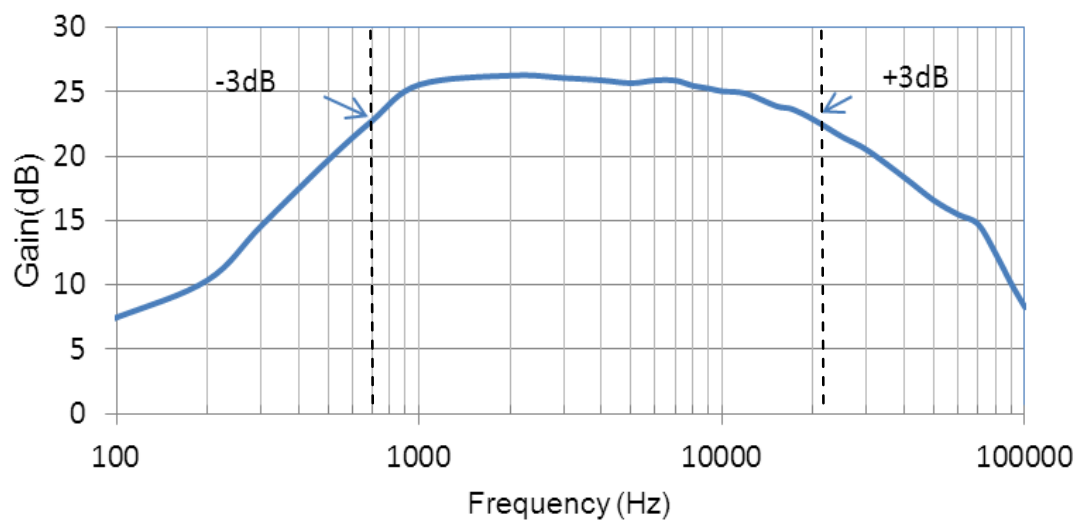

Fig. 3 The frequency response of TNU-SuperSID teaching module.

The flat frequency response is from $1 \mathrm{kHz}$ to $10 \mathrm{kHz}$ corresponding the gain of $26 \mathrm{~dB}$. The high pass filter of preamplifier keeps the frequency response low at frequencies below $700 \mathrm{~Hz}$ to remove the hum of noise from power lines.

\section{The System Settings and Data Collection}

The antenna is connected to the preamplifier, the sound card's driver and SuperSID software are installed, then preamplifiers is connected to the sound card to store data on a computer (Fig. 4).

The audio transformer with the wire ratio $1: 1$ is used to isolate the ground of preamplifier and the ground of the computer. The audio signals from the transformer go into the sound card, Sound Blaster's Audigy SE. The sound card has the A/D converter which digitizes the analog signal to transfer to PC. The A/D converter has the sample rate of $96 \mathrm{kS} / \mathrm{s}$ and 16 bit precision. The SuperSID software is used to record the sound files with extension "csv". The PC is connected to the Internet and its clock is synchronized by a time server with an accuracy of a few tens of milliseconds of UTC.

Fig. 5 shows the spectrum of $\mathrm{NWC} / 19.8 \mathrm{kHz}$, $\mathrm{JJI} / 22.2 \mathrm{kHz}$ and NAA/24 kHz signals at 12:42 UT on 31 May, 2014. On this spectrum, there is a broader hump visible in the $1,4 \mathrm{kHz}-27 \mathrm{kHz}$ range as a result of the radiation from lightning discharges. The NWC signals from Australia $\left(21.8^{\circ} \mathrm{S} ; 114.2^{\circ} \mathrm{E}\right)$ and NAA signals from USA $\left(44.38^{\circ} \mathrm{N} ; 67.16^{\circ} \mathrm{W}\right)$ are modulated with MSK (minimum shift keying) and JJI signal from Japan $\left(32.04^{\circ} \mathrm{N} ; 130.81^{\circ} \mathrm{E}\right)$ is modulated with FSK (frequency shift keying). However, JJI and NWC signals have seen clearly while, sometimes, the NAA signal disappears because of the effects of long path crossing both land and sea.

Fig. 6 shows the variation of VLF signals of JJI and NWC transmitters versus UT. It is obvious that the sunrise and sunset affect these signals with two dips. We can see spikes indicating the sudden enhancement 


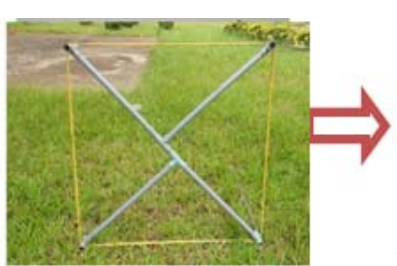

VLF antenna

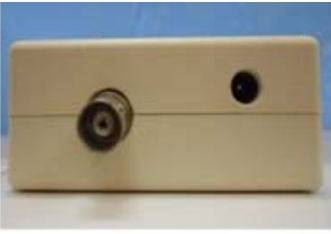

Preamplifier

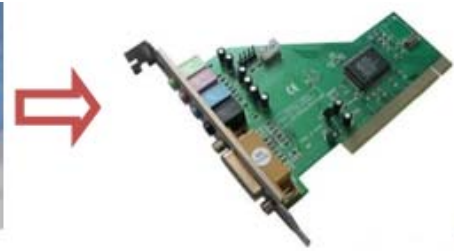

Sound card

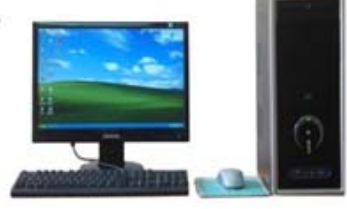

PC \& SuperSID software

Fig. 4 The system of TNU-SuperSID teaching module.

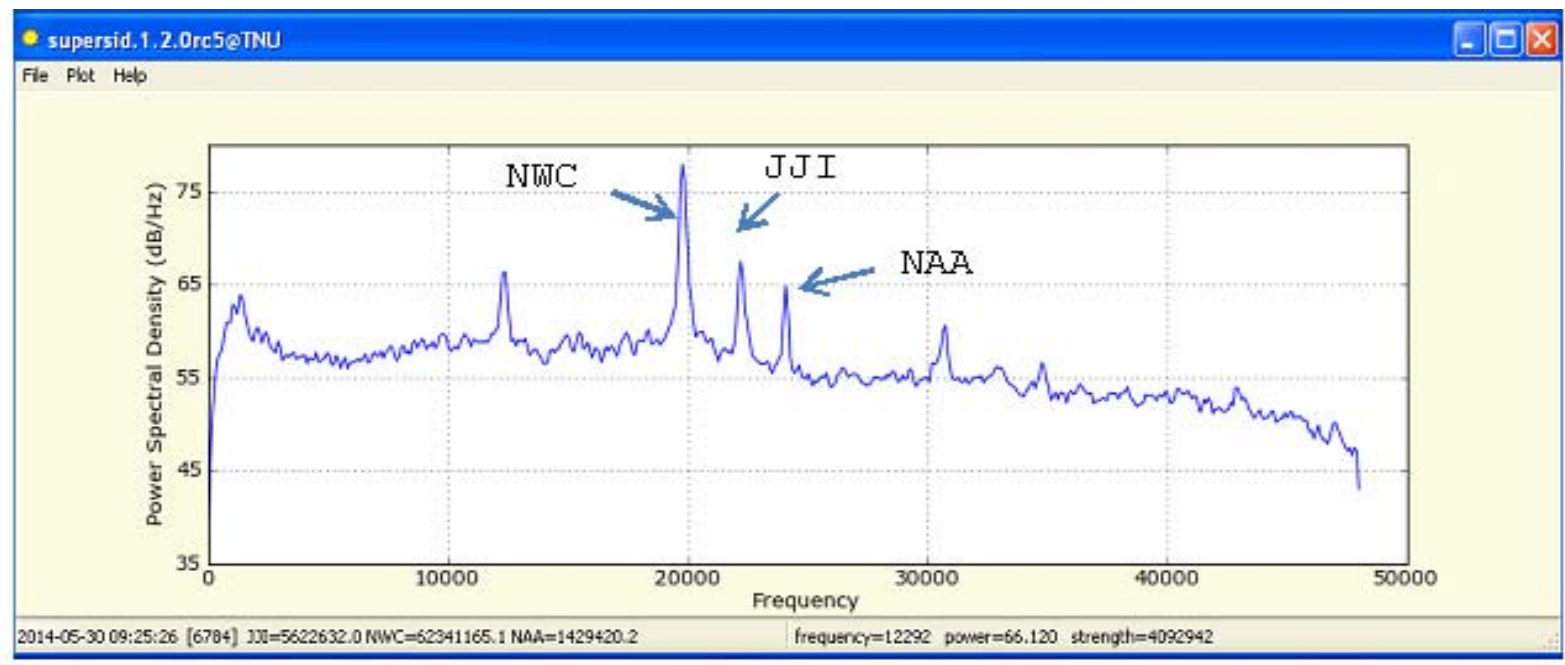

Fig. 5 Spectrum of 31 May, 2014. The spikes marked by arrows show the recorded VLF signals from transmitters.

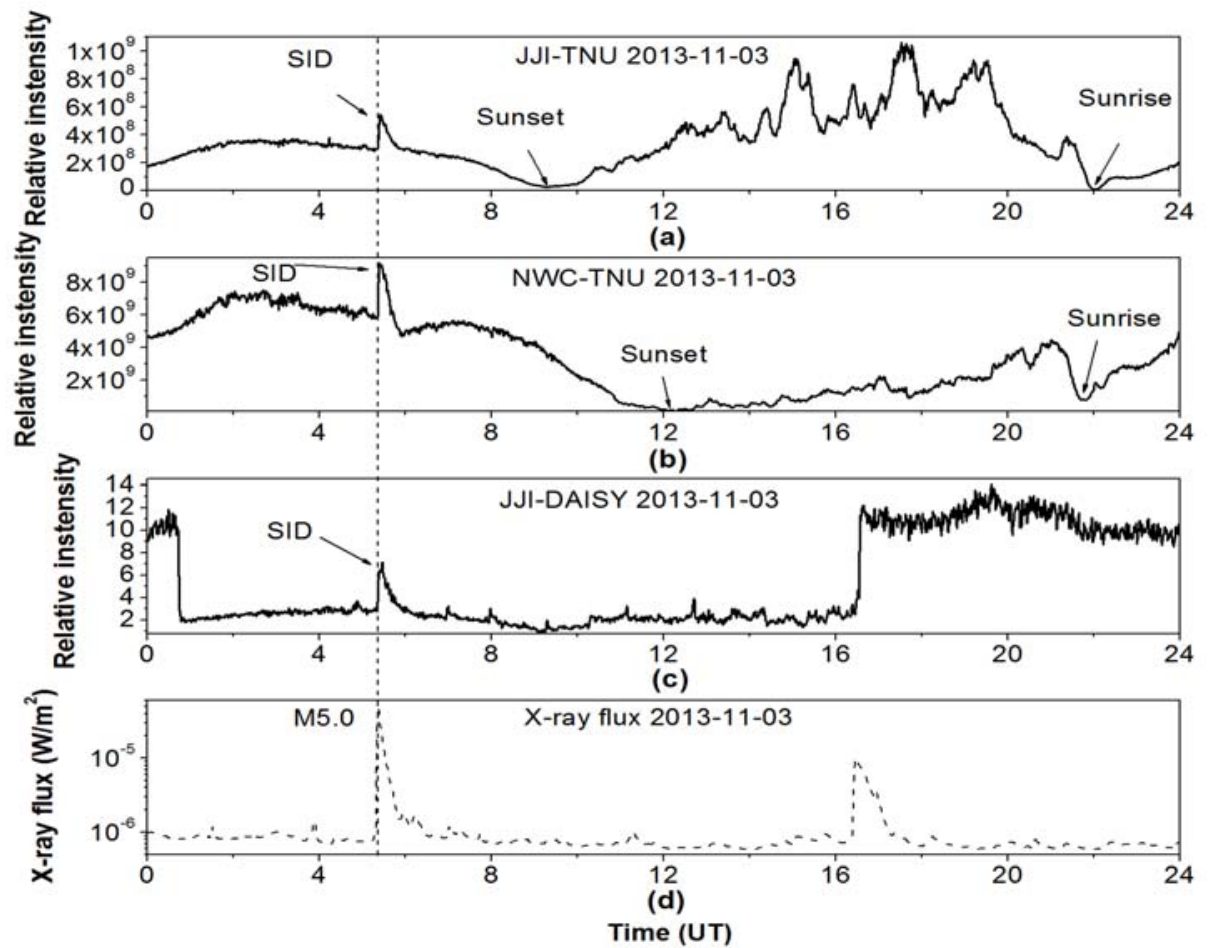

Fig. 6 The variation of VLF signal versus time universal time, UT (local time LT $=$ UT +7 ), and the recorded Sudden Ionosheric Disturbance due to solar flares with M5.0 class at 5:22 UT on 3 Nov, 2013. a), b) NWC and JJI signal recorded at TNU, c) JJI signal recorded at Daisy, Singapore and d) X-ray flux recorded by GOES. 
of VLF strength during strong solar flares with M5.0 class at 5:22 UT on 3 Nov, 2013. We also see the same case with JJI signal recorded by a SuperSID monitor at DAYSY, Singapore $\left(1.37^{\circ} \mathrm{N}, 103.8^{\circ} \mathrm{E}\right)$. The enhancement of $\mathrm{X}$-ray flux causes the increase and redistribution of the electron density of the D-layer. The upper boundary of the Earth Ionosphere waveguide becomes more sharp and the absorption of this layer is lower, and hence VLF strength increases [12].

\section{Educating Process}

In order to educate students, we explain the diagram of the VLF preamplifier circuit, operation principles of the antenna, concepts of space science and provide the students with user manuals. After that, the students are assigned specific tasks with different steps.

Step 1: Building antenna, assembling components on the PCB, and surveying the characteristics of the preamplifier.

Step 2: Connecting the preamplifier with antenna, installing the software and soundcard's driver, testing the receiver, recording the data.

Step 3: Comparing SID data recorded with GOES satellite data; comparing the reaction of different VLF signals recorded at other stations; determining the dips or spikes during the sunrise/sunset transition, discussing the results and explaining physics processes.

Step 1 and 2 can develop students' technical skills. Students can understand the function of low pass filter and high pass filter in the preamplifier when they survey the frequency response. With respect to step 3, communication skill, ability to work in groups and data analysis skill of students can be improved dramatically. By observing the real data, students will have a firm belief in the acquired knowledge of space science.

\section{Conclusions}

TNU-SuperSID teaching module has been developed at Tay Nguyen university with the collaboration of Stanford Solar Center of Stanford University. This module can observe the effects of sunrise and sunset phenomena and solar flares on the Earth's ionosphere via monitoring the VLF signals propagating from many transmitters to a receiver site. Students can participate in the project with different tasks: antenna construction and preamplifier assemble, system installation, data processing. Consequently, students can develop not only their technical, working in a group and communication skills, but knowledge of space science as well.

\section{Acknowledgments}

The authors would like to thank Stanford Solar Center and Society of Amateur Radio Astronomers of Stanford University for supporting us a SuperSID monitor and many helps. Special thanks to Prof. Dr. Deborah Scherrer for giving us the kind support. Many thanks to Dr. Tran Thanh Tung, vice dean of Faculty of Natural Science and Technology, Tay Nguyen university for encouraging us to accomplish our project.

\section{References}

[1] Scherrer, D., Rabello-Soares, M. C., and Morrow, C. 2007. "The Space Weather Monitor Project: Bringing Hands-on Science to Students of the Developing World for the IHY 2007 (Astronomy for the Developing World)." Proceedings IAU Symposium No. SPS5.

[2] Stanford Solar Center. 2010. "SuperSID Manual." Accessed January 6, 2010. http://solar-center.stanford.edu/SID/Distribution/SuperSI D/supersid_v1_1/Doc/SuperSIDManual_v1.pdf.

[3] World Meteorological Organization (WMO). 2009. "The Potential Role of WMO in Space Weather.”. Accessed 2009.

http://www.wmo.int/pages/prog/sat/documents/SAT-PUB _SP-5-TD-1482-Potential-Role-of-WMO-in-Space-Weat her-2008.pdf.

[4] Hunsucker, R. D., and Hargreaves, J. K. 2003. The High-Latitude Ionosphere and Its Effects on Radio Propagation. UK: Cambridge University Press, 640.

[5] Kivelson, M. G., and Russel, C. T. 1995. Introduction to Space Physics. Cambridge: Cambridge University Press, 
588.

[6] Lynn, K. J. W. 2010. "VLF Waveguide Propagation: The Basics, Propagation Effects of very Low Frequency Radio Wave.” In the 1st International Conference on Science with Very Low Frequency Radio Waves: Theory and Observation (AIP Conference Proceedings), 1286: 3-41.

[7] More, C. T., Sharma, A. K., Bhonsle, R. V., and Lynn, K. J. W. 2010. "Field Strength Measurement of VLF Radio Wave Propagation at $19.8 \mathrm{kHz}$ between Australia and India.” In the 10 th Australian Space Science Conference, 249-262.

[8] Grubor, D., Sulic, D., and Zigman, V. 2008. "Classification of X-ray Solar Flares Regarding Their
Effects on the Lower Ionosphere Electron Density Profile.” Ann Geophys 26: 1731.

[9] Mitra, A. P. 1974. Ionospheric Effects of Solar Flares. Holland: D Reidel, 294.

[10] Gustafsson, M. 2011. "Detection of Solar Flare Induced Ionospheric Perturbations on Narrowband VLF Transmissions." Thesis of Master, KTH School of Electrical Engineering, Stockholm, Sweden.

[11] Paschal, E. W. 1988. "The Design of Broad-Band VLF Receivers with Air-Core Loop Antenna.” Accessed May, 1988. http://traktoria.org/files/radio/antenna/VLF/.

[12] Grubor, D., Sulic, D., and Zigman, V. 2005. "Influence of Solar X-Ray Flares on the Earth Ionosphere Waveguide.” Serbian Astronomical Journal 171: 29-35. 